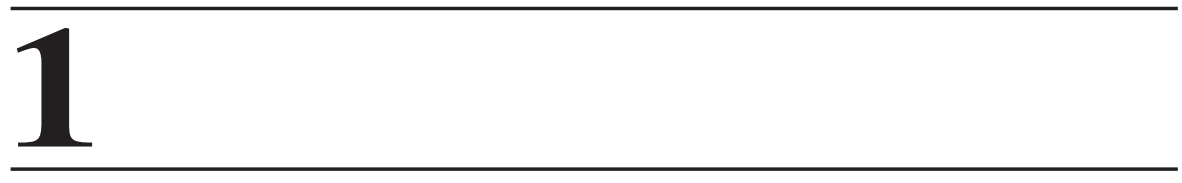

\title{
DOWNSTREAM PROCESSING OF MONOCLONAL ANTIBODIES: CURRENT PRACTICES AND FUTURE OPPORTUNITIES
}

\author{
Brian Kelley, Greg Blank, and Ann Lee
}

\subsection{INTRODUCTION}

Monoclonal antibodies (mAbs) are now established as the most prevalent class of recombinant protein therapeutics. They can be expressed at high levels in cell culture, are typically very soluble, and are relatively stable during processing. The nearly universal use of mammalian cell expression systems for $\mathrm{mAb}$ synthesis, combined with the selection of homologous, humanized $\mathrm{mAb}$ framework protein sequences, provides opportunities to harmonize manufacturing processes around base platforms that can then be used with only slight variations from product to product. In addition, by using a platform process, manufacturing plants designed for the production of one mAb can usually be readily adapted to produce others.

For these reasons, mAbs represent a unique group of biological products. They accommodate rapid process development time lines, can be produced in large quantities, and may be manufactured in multiple facilities during their lifecycle as a result of their common process flowsheets. As a result, they have relatively low manufacturing costs and benefit from the flexibility of production at either in-house or contract production facilities. Although mAbs are not commodity products that are substitutable in the clinical setting, they have

Process Scale Purification of Antibodies, Edited by Uwe Gottschalk

Copyright (C) 2009 John Wiley \& Sons, Inc. 
distinct advantages in production scale and cost, as well as in product development speed and convenience, when compared to other recombinant protein therapeutics.

This introductory chapter attempts to set the context for the following chapters, which cover many aspects of mAb purification in detail. A typical $\mathrm{mAb}$ purification process flow sheet is described and used to illustrate the impact of purification platforms on $\mathrm{mAb}$ production. Factors to consider with respect to the various process alternatives or new technologies described in upcoming chapters are addressed, emphasizing the integration of unit operations and process design principles into an optimized, holistic process. Both current practices and controversial topics are introduced, among them the challenges of very large-scale (VLS) production, issues related to facility fit, the maturation of process purification technology for $\mathrm{mAb}$ processing, the need for innovations in $\mathrm{mAb}$ downstream processing, and the impact of the evolving regulatory environment. It is hoped that this backdrop will stimulate critical thinking and comprehensive analysis when the processing options described in the following chapters are being considered.

\subsection{A BRIEF HISTORY OF cGMP mAb AND INTRAVENOUS IMMUNOGLOBULIN (IgIV) PURIFICATION}

The processes used for production of IgIV from human plasma differ from those used for recombinant mAbs. Figure 1.1 shows a consensus processing scheme, based on many published process flow sheets, for the purification of IgIV. Most IgIV processes lack chromatographic steps and instead rely on multiple fractional precipitation steps based on the Cohn process developed in the 1950s (1). Some recently developed processes include chromatographic

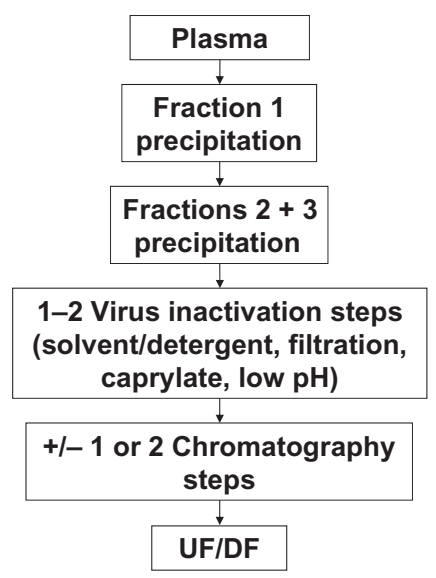

FIGURE 1.1 Cohn-based IgIV consensus process. 
steps, but this is used to a limited extent and still in combination with upstream steps based on the Cohn process $(2,3)$. The processes used for recombinant $\mathrm{mAb}$ purification have borrowed very little from plasma fractionation technology, other than ultrafiltration to formulate and to concentrate the drug substance. The low cost of manufacturing IgIV and the very large production scale have led to debate on the value of going "back to the future" and applying IgIV processing technologies to the production of recombinant mAbs. A review of current $\mathrm{mAb}$ processing platforms will put this proposal into context.

The first cGMP for mAb purification reflected the state of the art in the 1980s and early 1990s, prior to the accumulation of substantial process knowledge and the introduction of improved separation media that made today's more efficient and scalable processes possible. Examples of the diversity of early processes include the use of various microfiltration or depth-filtration media for harvest; affinity chromatography with Protein $G$ in addition to Protein A; conventional capture columns to protect the Protein A resin; incorporation of challenging separation methods for large-scale production, such as size exclusion chromatography (SEC); solvent/detergent virus-inactivation methods; and the requirement for four or even more chromatography steps (Figure 1.2). In addition, downstream processing was sometimes performed in the cold. Chromatographic media often provided relatively low loading capacities, which were not a significant issue when cell culture titers were measured in hundreds of milligrams per liter. To address the need for kilogram-scale production, very large bioreactors were used; the focus for capture resin selection was based on maximizing volumetric productivity and on the ability to

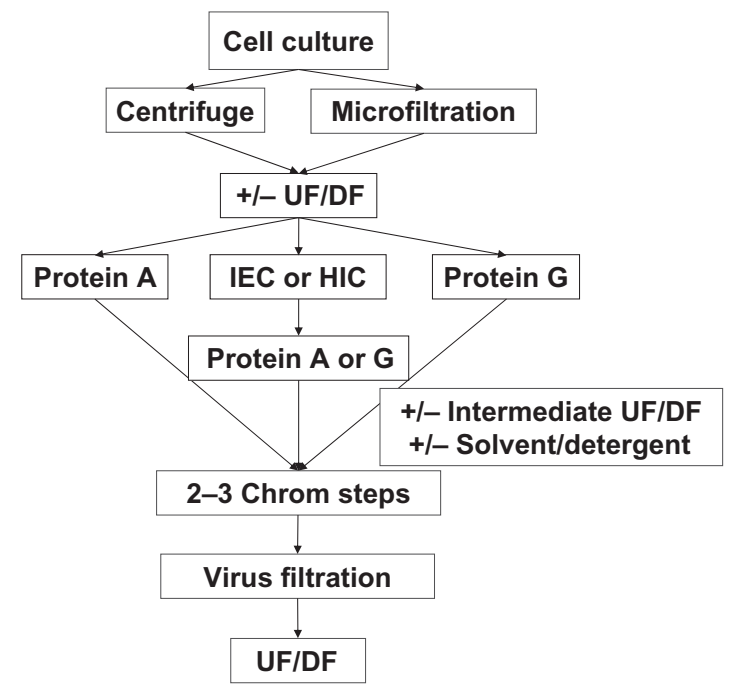

FIGURE 1.2 Early mAb purification schemes. IEC = ion-exchange chromatography; $\mathrm{HIC}=$ hydrophobic interaction chromatography. 
process large volumes of feed rapidly, rather than on the handling of large batches (greater than $20 \mathrm{~kg}$ of product). Many of these early mAb products were also derived from a more diverse set of framework protein sequences, reflecting the historical progression from murine and chimeric mAbs to today's fully humanized antibodies, which gave rise to a more varied set of process flow sheets.

\subsection{CURRENT APPROACHES IN PURIFICATION PROCESS DEVELOPMENT: IMPACT OF PLATFORM PROCESSES}

Despite the high degree of homology among humanized mAbs, variations in complementarity-determining regions and framework sequences make it difficult to define a truly generic purification process capable of processing many different $\mathrm{mAbs}$ without any changes to the operating conditions. Despite these variations, many companies have defined platform purification processes based on a common sequence of unit operations. A frequently used purification platform for mAbs is shown in Figure 1.3. The conditioned medium is first clarified by centrifugation, followed by depth filtration. Protein A chromatography offers direct product capture from the centrate and provides excellent purification and significant concentration of the product. The low-pH elution from the Protein A step also provides virus inactivation. Two chromatographic polishing steps are used to reduce host cell, medium, purification processrelated impurities and product impurities. Additional virus removal is usually achieved in these polishing steps. One of the polishing steps is almost invariably anion-exchange (AEX) chromatography, often run in the flow-through mode. The second polishing step is typically cation-exchange (CEX) chromatography, although occasionally ceramic hydroxyapatite or hydrophobic inter-

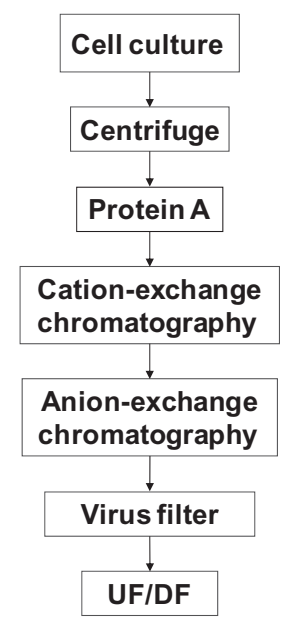

FIGURE 1.3 Typical mAb platform (current). 
action chromatography (HIC) are used. The remaining process steps include virus filtration (VF) and ultrafiltration/diafiltration (UF/DF) to formulate and concentrate the product, which is now the bulk drug substance. The efficiency, robustness, and scalability of this standardized process have resulted in the rapid convergence of process development groups in the industry around a similar process flow sheet $(4,5)$.

The establishment of platform processes for $\mathrm{mAb}$ production has already had an enormous impact on process development strategy and activities, and is just beginning to affect the world of commercial manufacturing. At this point, very few companies have two or more commercial mAbs that are purified by a common platform process. Many mAbs currently in the clinical pipeline, however, are manufactured by a process similar to the standard process shown in Figure 1.3. The gradual progression of these early-stage processes from clinical to commercial production enables additional efficiencies in production that will reduce the costs of goods (COGs) and accelerate responses to surges in product demand. The benefits of efficient facility management (e.g., reductions in changeover time and the use of common raw materials and equipment) and flexible commercial production (e.g., balanced production schedules among multiproduct facilities) will be realized more slowly than the gains seen today with product candidates in the early stages of clinical development. The combination of platform processing, multiproduct facilities, rapid product changeover, and flexible sourcing between contract manufacturing organizations (CMOs) and in-house production facilities achieves an industrialization of $\mathrm{mAb}$ production that will be unprecedented in the field of recombinant protein biologics. Antibodies could become a class of therapeutic biologics that support the treatment of large patient populations while remaining cost competitive with small molecules. To achieve this vision, the biopharmaceutical industry must take advantage of the opportunities presented by the ease of development, validation, and production afforded by platform processes.

Given the value and broad adoption of processing platforms, combined with an installed production facility base designed for them, there is enormous pressure to conform to such platforms with future products. As a result, options for unit operations, raw materials, step sequences, control systems and algorithms, and processing equipment are limited. While these restrictions may at first seem highly constraining, they require other challenges to be addressed, e.g., the establishment of highly efficient work processes that rapidly define the appropriate processing conditions for each new $\mathrm{mAb}$ that enters the pipeline, as well as the definition of a common set of optimization approaches and process characterization studies that will streamline late-stage development of clinical products.

Clarification operations such as centrifugation often vary little from product to product, provided that the cell culture process is not radically different. Large changes in the cell concentration or viability in a bioreactor will affect clarification, but provided that the unit operations are designed for the worst- 
case feedstream, few if any modifications will be needed for new mAbs. The capacity of centrate depth filters can vary significantly, depending on feedstream, and should be optimized for robustness while the costs of raw materials are minimized. Similarly, the platform's ultrafiltration steps (VF and final ultrafiltration) should be largely unaffected by the change in the mAb. The unit operations that are most likely to require tuning are the chromatography steps. Even there, the standardization of many elements in a chromatographic unit operation will streamline development time lines by focusing on key factors influenced by product characteristics (5). Process variables that are often specified for platform processes include resin and membrane selection, column bed height, wash volumes, loading capacity, membrane flux, and target bulk concentration. This effort simplifies and accelerates early-stage process development.

The Protein A capture step is generally a very robust operation that can tolerate changes in bioreactor harvest conditions and product characteristics (see Chapter 4). The variables that may be influenced by product or feedstream variations are dynamic binding capacity, the optimal composition of the column wash solution, and the elution conditions. Variations in these process parameters arise from differences in the affinity of Protein A for the $\mathrm{mAb}$, the steric hindrance among molecules (6), and variations in impurity levels and species in the feedstream, probably caused either by the cell line and bioreactor management or by the properties of the mAb itself.

The most common variables for the ion-exchange polishing steps include the column-loading and solution compositions (e.g., $\mathrm{pH}$ and counterion concentration) and the wash and elution compositions. In some cases, there can be major changes to the platform, as when a highly acidic mAb has strong affinity for an AEX resin and the typical flow-through operation must be abandoned in favor of a bind-elute step. Broad ranges in the affinities of ionexchange resins have been described $(5,7)$ and highlight one area where the diversity of $\mathrm{mAb}$ properties has an impact on the purification process.

Given that chromatography variables may be optimized independently for each $\mathrm{mAb}$, there are choices to be made regarding the investment in earlystage process development. Two extremes are represented by tailored, as compared to generic, processing conditions. An example of the tailored approach is the use of high-throughput screening to define unique operating conditions for each mAb, e.g., to optimize an AEX polishing step (8). Similarly, scouting studies using gradient elution for bind-elute steps could be used to tune the elution conditions for a CEX step. The generic approach to process development would use a single $\mathrm{pH}$ for the AEX step and adjust the load counterion concentration only by dilution. This minimum counterion concentration would vary from $\mathrm{mAb}$ to $\mathrm{mAb}$; an even more flexible step would use a single counterion concentration, which allows successful processing of the majority of mAbs without significant product losses of the most acidic family members. Likewise, in a generic approach for CEX, the step could be designed with a very low conductivity for the load combined with a broad gradient 
elution, which could potentially encompass successful processing of a large number of mAbs (see Chapters 5-7, which provide examples of this approach). The trade-offs of the two approaches would be influenced by the interplay of process development resources and time (more for the tailored approach, less for the generic), manufacturing efficiency (higher for tailored, lower for generic), and other factors, such as $\mathrm{mAb}$ characteristics.

After Phase I process development, companies typically engage in at least one additional cycle of process development (commonly termed late-stage development), which defines the Phase III process and is subsequently used for product launch. This second cycle often involves changes to the cell culture process, including media reformulation, changes to the feeding strategy, optimization of culture duration, and potentially the introduction of a new cell bank or cell line. Although changes to the purification process during this second cycle are unlikely to have the same regulatory impact as cell line changes, the elimination of a step (e.g., the elimination of one of two polishing steps) could cause a problematic change in an impurity profile. Given that several years may have passed between the development of the Phase I and Phase III processes, consideration should be given to upgrading the purification process to include the superior separation media that have been recently introduced to the market. Additional optimization studies will define the final process control ranges for key and critical process parameters and will investigate the processing parameters that are unique to each mAb (e.g., column capacities, resin and membrane lifetimes, in-process hold times, and maximum bulk concentration).

If the implementation of radically new processing technologies is considered, this option is typically weighed at the late-stage development cycle rather than at the early stage. Alternate, off-platform technologies (9) would require sustained and significant effort to define processing parameters; establish process robustness; acquire and test novel raw materials; and specify, purchase, and validate new equipment. These factors strongly support an approach where implementation of new technology on a Phase I time line would be used only if the new process technology represented the sole means of enabling clinical production. The "speed-to-clinic" driver would almost always trump the benefits in COGs or the productivity benefits that novel technology would offer at this stage of development. This would force novel technologies to be considered during the next two cycles of process development, either for the Phase III definition or as a post-licensure change.

This argument may appear to establish a conundrum for the introduction of novel, off-platform unit operations. If they will not be introduced in Phase I processes due to speed-to-market pressures, they must be delayed to the Phase III process. Yet the introduction of significant process changes (especially those that could negatively affect the impurity profile of the product) after Phase I safety studies is a high hurdle that may require additional clinical trials, increasing the cost of the drug development and potentially delaying product launch. The introduction of new technology after 
licensure may appear to have an even higher bar, even if the risk of an untoward effect of process changes is very low. There have been instances of significant post-licensure changes in purification processes for recombinant proteins (10-12), which in some cases required additional clinical studies. The U.S. Food and Drug Administration (FDA) allows process changes to be managed under comparability protocols without clinical trials if the process change has no impact on product safety, potency, or efficacy, and if the product is well characterized.

A major benefit of establishing a purification process platform is realized when a reasonably long period elapses without significant changes that alter process development or manufacturing operations. This leads to an approach whereby process improvements are bundled and introduced through a controlled, internal review and decision-making process (a "punctuated equilibrium" for the platform). The evolution of a company's platform is a healthy process, as superior separation media and accumulated development knowledge combine to offer significant advantages in development speed, reduction in COGs (see Chapter 9) or improved plant productivity. The change-control process for the platform should be managed to prevent changes from occurring too frequently, while allowing the introduction of clearly superior technology when appropriate.

\subsection{TYPICAL UNIT OPERATIONS AND PROCESSING ALTERNATIVES}

Centrifugation is currently the method of choice for harvest operations (13, 14). Continuous-flow disk-stack centrifuges are robust, are easily scaled across various fermenter volumes, and use a relatively generic set of processing parameters. It is unlikely that centrifugation can provide a sufficient degree of solids removal to allow the centrate to be processed directly by the initial capture chromatography without further filtration, although processing alternatives such as flocculation may offer a significant reduction in the filtration area required (15). Depth filters may remove protein [host cell protein (HCP) or product] during filtration (16), and changes to filtration media should be evaluated carefully. Centrifugation parameters can affect the filter area needed and can influence the colloidal properties of the feedstream. Therefore, consideration of both centrifugation and filtration parameters is required for optimal performance and integration of these steps. Although centrifugation is likely to remain the preferred choice for large-scale operations, smaller-scale cell culture harvests $(\leq 400 \mathrm{~L})$ can also be performed using a combination of depth and size filtration. Newer technologies, such as pod harvest units, make filtration a more attractive option at small scales.

The majority of processes use Protein A chromatography as the initial capture chromatography step. Two potential issues associated with Protein A 
chromatography are leaching of the Protein A ligand from the resins into the product pool and the higher cost of Protein A resins as compared with nonaffinity resins. Alternative ligands to Protein A that are either small molecules or polypeptide mimetics of Protein A have generally not delivered increased load capacity while maintaining selectivity (17). Most of these resins are not known to be under active investigation in industrial laboratories. Other alternatives to Protein A chromatography as a capture step typically include ionexchange chromatography, and because many mAbs have pIs greater than 7, CEX resins are the primary choice (see Chapter 5). With CEX, the $\mathrm{pH}$ and/or conductivity of the harvest cell culture fluid may need to be adjusted, and in some cases the product in the feedstream should be concentrated using ultrafiltration, to obtain optimal capacity and throughput. This conditioning operation should be evaluated when the overall costs of affinity capture are compared to those of nonaffinity capture. The lower $\mathrm{pH}$ and conductivity may affect antibody stability in the feedstream (because of acid-activated proteases) or precipitation of proteins (either product or HCP), thus necessitating increased filter area. However, precipitation of HCP during conditioning may contribute to HCP removal during the process (see Chapter 5), although it could cause complications during ultrafiltration. The potential of impurity precipitation as an up-front conditioning step is addressed later (see Chapter 15), although the economics of the technique do not appear to be favorable, at least at the high concentrations of the precipitant evaluated by those authors.

The number and types of polishing steps will be determined by the nature of the product and process-related impurities. In general, a Protein A pool will be more pure than a nonaffinity pool. Cell culture conditions that increase expression but also result in higher levels of aggregate or charge variants may influence the choice of affinity or nonaffinity process or may require additional polishing steps. A nonaffinity process is generally less amenable to a platform approach because not all antibodies bind well to the capture column without custom feedstream modifications, as in the case of CEX resins. Furthermore, a Protein A capture step offers greater freedom to match the subsequent processing step without feedstream adjustments (see Chapter 4). If the affinity pool is relatively pure, only one additional chromatography polishing step may be necessary. There have been platforms that use just two chromatography steps, where the AEX step is run under conditions of weak partitioning (8), as shown in Figure 1.4. Two-column processes offer advantages beyond the obvious cost reduction associated with eliminating a step from the process (see Chapter 5, which provides examples of processes comprising two columns plus a membrane adsorber) New, mixed-mode chromatography resins may also aid in minimizing the number of polishing steps by increasing the separation performance for each resin (see Chapter 6). Product-related impurities with only slight charge or size differences can be the greatest challenge for the purification process because of their similarity to the product. Controlling these impurities to acceptable levels during the cell culture process facilitates a two-column process. 


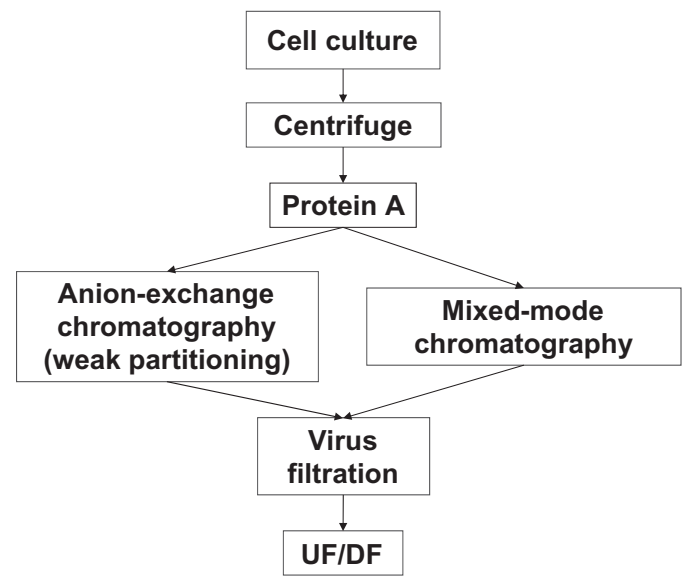

FIGURE 1.4 Emerging two-column platforms.

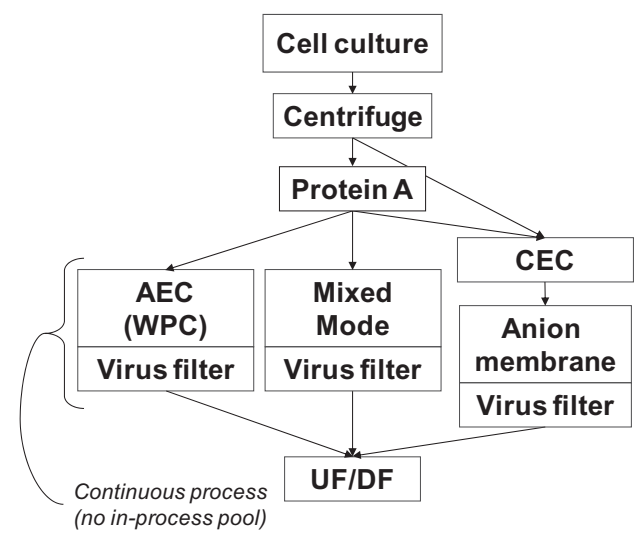

FIGURE 1.5 Future high-efficiency platforms. AEC = anion-exchange chromatography; $\quad \mathrm{CEC}=$ cation-exchange $\quad$ chromatography; $\mathrm{WPC}=$ weak $\quad$ partitioning chromatography.

Future platforms could build on continuous processing, two-column processes, and alternative unit operations (Figure 1.5). Alternatives to chromatography may simplify processes, reduce costs, and facilitate the processing of large batches. Currently, binding capacities for adsorptive membranes are lower than those of resins, making them most suitable for flow-through chromatography operations in which relatively low amounts of impurities are bound. Membrane adsorbers could replace an ion-exchange polishing step and may offer reduced buffer consumption and small pool volumes when compared to resin column chromatography (18). They may also be more conducive 
to a continuous process in which the feedstream moves through each unit operation without the collection of a discrete pool, although $\mathrm{pH}$ and conductivity targets must coincidently match subsequent unit operations. Other alternative technologies include crystallization and precipitation (9) (see Chapter 14). For individual antibodies, these technologies may provide a performance benefit in cost or productivity that would merit their implementation in a process. However, because of mAb variability, they are not likely to be amenable to a platform process, and they require additional resource investment during process development. Factors to consider in evaluating such novel process options include annual production requirement, expression level, COGs target, and production costs. As outlined later (see Chapter 12), economic considerations must include capital investment, operating expenses, economies of scale, the impact of opportunity costs of extended changeover periods, and the processing success rate.

The concentration of drug substance is linked to the requirements for drug product. For intravenous delivery, the drug product concentration may only be $5-20 \mathrm{mg} / \mathrm{mL}$. Drivers for concentrations of $100 \mathrm{mg} / \mathrm{mL}$ or greater include the benefits of subcutaneous administration and a reduction in the number of drug substance storage vessels (often large stainless steel tanks) for very large batches. Achieving concentrations greater than $100 \mathrm{mg} / \mathrm{mL}$ depends on the solubility of the antibody as well as the formulation excipients, concentrations, and $\mathrm{pH}$. Operational challenges to high-concentration formulations include $\mathrm{mAb}$ stability during concentration and diafiltration, product losses due to system hold-up volume and incomplete recovery, and the extreme viscosity of some antibodies as the concentration increases. These challenges may be addressed by careful equipment design and by a formulation that minimizes viscosity and maximizes product stability.

\subsection{VLS PROCESSES: TON-SCALE PRODUCTION AND BEYOND}

The recent increases in cell culture titers are likely to continue as cellular productivity and cell density improve and as production phases are extended. Titers as high as $10 \mathrm{~g} / \mathrm{L}$ have already been reported (19), and titers of $2-5 \mathrm{~g} / \mathrm{L}$ or more will be common. The rise in titers combined with predictable scale-up to larger bioreactors has sparked debate about purification bottlenecks that limit a production plant's capacity. However, efforts to intensify the purification process should stave off capacity limits for all but the largest-volume products $(20,21)$. The benefits of chromatography resins and ultrafiltration membranes with elevated capacities and improved volumetric productivities allow purification processes to keep pace with advances in cell culture. The most common limitations are buffer make-up and storage capabilities, in-process pool tank volumes, and the scale of the production equipment. High-capacity resins and membranes reduce buffer consumption, generate 
high-concentration in-process pools that minimize process volumes, and allow larger batches to be processed with existing equipment. A two-column process is significantly more productive than three-column or four-column purification trains, and for almost all products, it should enable VLS production without constraint.

Increased cell culture titers generated by extended fed-batch operations and high cell densities give rise to very large batch volumes (e.g., a $10 \mathrm{~g} / \mathrm{L}$ titer in a 25,000-L bioreactor would generate $250 \mathrm{~kg}$ of harvest material and a $200-$ $\mathrm{kg}$ batch of drug substance). An extremely long bioreactor production phase affects facility utilization and staffing. At this scale, very few batches would be required to supply the market for most mAbs. A 1-ton demand would be met with only five batches, requiring very little production time in a plant operating multiple bioreactors. Efforts to level the staffing load or to manage product inventory may result in the operation of production bioreactors at less than maximum volume, if plant capacity is not appropriately matched to demand (this constraint is relaxed if the mAb is produced by a CMO). Furthermore, as the bioreactor production stage lengthens, the mismatch between the shorter cycle of the downstream process and the bioreactor becomes more problematic (22). Harvesting a single reactor for an extended culture (e.g., every $24 \mathrm{~d}$ or more) and using a 3-d purification process means that the purification staff may be unoccupied for a large portion of the interval between harvests. While this ratio allows a single purification train to service eight bioreactors, the enormous production capacity of a plant running high-titer processes would invariably result in inefficiencies unless the plant is also making other products.

Purification costs are the dominant drivers for VLS processes, but they are still low when expressed on a per-gram basis. Key COG components are the Protein A resin and the virus filter. Currently, the reuse of high-capacity Protein A resins for 200 cycles or more (23) reduces costs associated with this unit operation to approximately $\$ 1$ per gram. This analysis presumes that the resin is used to its full lifetime, which will be the case for large-volume products in steady production over several years. When demand does not require enough runs to extend the Protein A resin to its validated lifetime, COGs will increase, with the cost per gram declining over time as the initial investment in the resin is diluted over successive batches (e.g., if a 100-kg annual demand can be produced with two $50-\mathrm{kg}$ batches, each requiring five loading cycles for the Protein A step per batch, the resin will not reach 200 cycles of use until 20 years after launch, so limits on chronological resin age almost certainly dictate a shorter life span). Another significant purification cost is the VF filter. Provided that the membrane can offer reasonable fluxes with highly concentrated feedstreams, an extended operation of several hours will minimize the necessary membrane area and the cost. The reuse of VF membranes is attractive, especially for new membranes that are developed to withstand harsh sanitization cycles and do not require destructive post-use integrity testing. 


\subsection{PROCESS VALIDATION}

Process validation of purification processes defines process parameter ranges, critical process parameters, virus removal, membrane and resin lifetimes, and process pool stability. Control ranges ensure that the goals of the unit operation, including the appropriate product quality, are met. The variability of the incoming feedstream, the capability of the downstream process steps, and the potential interactions of all parameters for each step must be considered. A chromatography step may have 10-20 operating parameters. A series of designed experiments in which all parameters are examined would result in an impossibly large study. By applying an understanding of the process, along with a risk assessment, parameters can be placed into two categories: those that may have interactions with other variables and those that can be studied in singleparameter studies. For an ion-exchange column, examples of the potentially interactive variables include elution $\mathrm{pH}$, elution conductivity, column loading, and temperature. Single-parameter studies might include flow rate, bed height, wash volumes, and pooling conditions. The risk assessment should contain the rationale for the type of study chosen for each parameter.

The process platform also offers significant benefits during process characterization and validation. Modular validation leverages data from previous studies to support the control ranges for a new antibody (24). The criteria that allow a modular approach should be defined, as should the parameters that qualify for modular or product-specific validation. For example, if a study for antibody X had shown that Protein A bed heights between 15 and $25 \mathrm{~cm}$ had no effect on the process, that study could be used to support the same bedheight range for antibody Y. However, because different antibodies have different dynamic binding capacities on Protein A resins, a product-specific study for the load range would be necessary. Unit operations that tend to be less product specific, such as centrifugation, virus filtration, low $\mathrm{pH}$ virus inactivation, and UF/DF, are the most amenable to the modular approach.

The quality by design $(\mathrm{QbD})$ initiative is a comprehensive approach to product development and life cycle management in which the product and process parameters are designed to meet specific objectives. A thorough understanding of process parameters and their interactions and impact on product attributes defines the design space (25). Once approved, this design space offers regulatory flexibility for post-approval process changes. Validation studies must address the input variables (e.g., impurity levels) to the unit operation from the preceding step. Modular validation complements QbD and the definition of design space for platform processes. A company can use data from validation studies on similar molecules, combined with risk analysis, to define the design space for a related molecule. As a company accumulates data on several mAbs, it may be possible to develop a master file that provides the foundation for the design space for each new $\mathrm{mAb}$ manufacturing process.

For mAbs produced in mammalian cells, validation of the purification process to remove or to inactivate adventitious viruses is a requirement (see 
Chapter 8). In many cases, the platform processes for mAb manufacturing have an exemplary viral safety profile: they use cells, such as Chinese hamster ovary cells, which do not express viable retroviruses (26); use a culture medium with no animal-derived raw materials; include viral safety tests and procedures for postproduction cells for each lot; and establish the capability of the purification process to clear or to inactivate viruses. The nature and scope of these studies-including the number and types of viruses studied, determination of the overall clearance of the model viruses, and confirmation of the validity of the scale-down model and of the ability of resins to remove virus over their claimed lifetime-are described in the International Conference on Harmonisation Q5A (27). The concept of modular viral validation was first established by the FDA in 1997 (28). It is critical to establish criteria for the use of modular validation data. As an example, for a chromatography step, these criteria should include the exact resin type, its position in the process, the robustness of performance, and the comparability of process parameters (e.g., flow rate, protein load, and bed height).

An understanding of the mechanism of virus removal or inactivation is an important element of the modular approach. The design space for a step is established by assessing the effect of process parameter ranges on product purity (e.g., product variants, aggregates, and $\mathrm{HCP}$ ). If a step contributes to viral safety, an understanding of how these parameter ranges affect virus removal is also needed. However, virus removal validation studies are complicated and expensive. Therefore, studies are performed at the set point for process parameters. To address this issue, modular data from a comprehensive study on the effect of process parameters on viral clearance performed for one $\mathrm{mAb}$ (to define its design space) are used to support the design space for subsequent mAbs. With the mechanism of action and process robustness established, companies may make modular claims for $\mathrm{pH}$ inactivation and VF (29). Typically, the other step that contributes to viral safety is AEX chromatography. If this step is amenable to modular claims, then there may be no need to conduct product-specific viral clearance studies for clinical studies if the extent of viral clearance from all modular studies is great enough to provide a sufficient safety factor.

Even when modular validation can be applied, unique studies will be required for each $\mathrm{mAb}$. The hold times for conditioned media and in-process pools will be a unique combination of the feedstream, impurities, and the $\mathrm{mAb}$ sequence and structure. Forced decomposition studies should be performed to identify degradation pathways. Resin reuse studies are unlikely to be amenable to a modular validation approach because resin lifetime will be dictated by unpredictable effects of the antibody's properties as well as the impact of feedstream variability arising from the unique characteristics of individual cell lines. 


\subsection{PRODUCT LIFE CYCLE MANAGEMENT}

Whereas the preapproval process development phase typically lasts 5-7 years, the post-approval commercial manufacturing life cycle may extend to 20 years or more. The manufacturing life cycle includes post-approval process changes, transfer to new manufacturing sites, and possibly process scale-up. The breadth and depth of process development, characterization, and validation can greatly facilitate these changes. The reasons for making post-licensure changes are varied, but the most common motivation is to increase production capacity to meet increased demand. The approaches to increased capacity take a variety of forms and present different technical and regulatory challenges. Transferring the process to a new manufacturing facility at a similar scale is the most straightforward approach, but if the second facility is not an exact fit for the process, adjustments may be necessary. Changes in scale are usually associated with a facility change. If the scale-up of the cell culture and purification processes is not aligned, particularly if the purification equipment is undersized relative to cell culture, then it is likely that process adjustments will be necessary.

The interval between the definition of the cell culture and purification processes and product approval may be several years. During this time, advances in cell culture technology may enable increases in titers of $50 \%$ or more, which could be used to increase productivity of current products through postlicensure process changes. The effects of this increased titer on the purification process and facility are far-reaching and will probably require both process and equipment changes. If no changes are made to the operating conditions of the purification process, direct scale-up requires increases in column diameter and volume, membrane and filter areas, flow rates, and buffer and pool volumes. For facilities designed several years prior to the process improvement, the maximum titer used as a basis for the plant design is typically lower than that provided by the newest product.

Before process changes are made, a thorough assessment of the locations of purification bottlenecks is necessary. Changes to equipment, although perhaps more straightforward from the technical and regulatory perspectives, may be constrained by space availability, plant downtime during retrofitting, and capital expense. Purification process changes fall into two general categories. The first category includes modifications to optimize wash and elution volumes, to narrow pooling conditions, and to increase column capacities. How the ranges for these parameters are specified in the license will determine the regulatory pathway to the implementation of these changes. The second category of changes involves substantial modification to the process and requires regulatory approval prior to implementation. High-capacity chromatography resins can be used to address process bottlenecks. Pool-volume reduction affects both the step's pool volume and the downstream pool volumes if that step is operated in the flow-through mode. Because one main facility limitation is tank number and volume, changing the order of unit operations to minimize 
the effect of $\mathrm{pH}$ and conductivity adjustments for downstream steps can be beneficial. Of course, adding UF/DF steps is a one way to address tank limitations, but this change involves new capital equipment, downtime, and space considerations.

Increases in the product titer may not be the only changes to the feedstock. Modifications to the cell culture process may also change the product and process impurity profiles. Other changes to the feedstock include increased debris load with different physical properties, which will challenge the harvest operation. Evolving regulatory requirements are also drivers for process changes. These regulatory drivers may not be limited just to requirements of the national and international regulatory agencies [e.g., the FDA and European Medicines Evaluation Agency (EMEA)]; they may also include state and local agencies that govern areas such as hazardous waste and discharge into the local water system.

Risk assessment should assess the scope of process characterization work needed to support process changes. This assessment should evaluate the impact of the changes on the overall process and the applicability of validation studies from the original process. For example, if changes were made only to the cell culture process, the Protein A step would need to be revalidated. If the Protein A pool from the new process was comparable (i.e., had the same level of product and process impurities), then the unit operations downstream of Protein A would not need to be revalidated.

Commercial manufacturing is critical to the patients who rely on these drugs, and uninterrupted product supply is dependent on the availability of raw materials. For many purification raw materials, such as chemicals, supply is not critical because multiple suppliers can meet the necessary quality and quantity requirements. However, for some raw materials (e.g., chromatography resins), similar raw materials are not interchangeable among vendors. During process development, a specific resin is chosen for each step and the process optimized for that resin. Changing to a similar resin may not result in the same purity and yield under the same operating parameters. Certain membranes and filters may require unique operating conditions. For these processspecific raw materials, it is critical to have a risk-mitigation plan that will ensure uninterrupted manufacturing should a vendor be unable to supply a raw material (30). The risk can be mitigated by carrying an additional inventory of these raw materials or by developing processes with alternative raw materials. When alternative raw materials are used, the product quality of the drug substance must still meet the appropriate specifications. Ideally, an alternative raw material will provide the same process performance as the original raw material, although other factors (e.g., cost, ease of use, and process robustness) may make it a second choice. If the alternative raw material does not result in the same product quality in the intermediate process pool, it will be necessary to assess whether downstream steps can achieve the same product quality either with or without modification. Once alternative raw materials have been identified, an implementation strategy should be developed. The 
cost of the work necessary to develop, characterize, and validate an alternative raw material should be balanced against the cost of carrying an additional raw material inventory. Other factors to consider include the resin's lifetime, cost, and annual demand. When a raw material is used in more than one process, cumulative use and business risks also need to be considered. A platform process that relies on a small number of raw materials minimizes the number of raw materials in inventory and may reduce the overall backup required. However, this approach increases business risk, because a supply disruption may affect many products.

Raw materials may be grouped into classes such as chromatography resins, sterilizing filters, virus filters, and ultrafiltration membranes. The degree to which such raw materials can be substituted in these classes can be considered according to their intended use. For example, there may be significant differences among CEX resins in terms of resolution, with each resin requiring distinct operating conditions. These differences are based on the unique physical (e.g., bead and pore size) and chemical (e.g., ligand and resin matrix) properties of resins. Alternatively, sterilizing filters have a clear mechanism of separation (a size differential), and the operating parameters or design space can be defined to cover various filters. Taking this a step further, as the QbD concept evolves and design space is increasingly defined on the output of a unit operation, it may be possible to use different raw materials for a step as long as the output of the processing step (e.g., product purity and isoform distribution) is maintained.

For post-approval process changes, $\mathrm{QbD}$ and design space can greatly facilitate such changes. Knowledge gained during process development leads to a thorough understanding of process input ranges and process outputs, particularly for critical quality attributes, and thus lays the foundation for a robust design space. Such a design space is the basis for consistent, reproducible manufacturing operations, but also allows changes within the design space over the product's life cycle in response to changing conditions.

\subsection{FUTURE OPPORTUNITIES}

With standard mAb purification platform processes firmly entrenched in the industry and representing the current state of the art, one might ask what the future will hold. Several opportunities addressing this question will be presented in this section. Some of these suppositions may come to pass, while others may never be adopted.

Because mAbs are a group of compounds large enough to warrant consideration as a separate class (28), the adoption by regulatory agencies of common targets for impurity levels could be of great value to the industry. These targets would define process development design principles that are generally regarded as safe and would represent impurity levels that are readily achievable with standard processes. Currently, the only impurity that has a well-accepted limit 
is host cell DNA. The FDA initially issued guidance that specified $10 \mathrm{pg} /$ dose (31), and then $100 \mathrm{pg} / \mathrm{dose}$ (28), and this was followed by World Health Organization guidelines that specified $10 \mathrm{ng} /$ dose (32).

At the 2003 Well Characterized Biologicals conference, FDA representatives cited typical levels of HCP, aggregates, and residual Protein A levels for mAbs described in Phase I Investigational New Drug Applications (33). Although levels of process- and product-related impurities have not been translated into regulatory guidance documents, the presentation suggests these levels are generally regarded as safe with the caveat that dose, dosing frequency, and route of administration are all important factors have not been issued in regulatory guidance documents, and while they do not reflect a common international position, the presentation of these levels hints at the possibility of establishing limits that are generally regarded as safe for these host cell and process-related impurities. Clarity regarding these targets could greatly simplify process development, enable more productive processes that do not "overdevelop or overengineer" without compromising product safety, and aid in the regulatory review of both clinical filings and commercial license applications.

Of course, the appropriate limits on impurity levels should be evaluated on a case-by-case basis, with risk adjustment for therapeutic dose, disease indication, dosing frequency, and other clinical factors. Ideally, a set of common target impurity levels would cover the most conservative case, so that adjustments would generally be made only to increase the impurity levels if warranted by process capability, following a thorough review of the clinical context for the product. In the opposite case, companies would be expected to recognize the highly unusual combination of factors that would warrant a reduction in the target impurity level in order to lower the risk of adverse clinical events.

Programs are under development at several companies for identifying, early in the discovery phase, mAbs that have inherent processing or quality issues, such that mAbs directed at the same target but with alternate sequences (and thus improved characteristics) could be chosen instead. Examples of the product attributes that may be included in a manufacturing feasibility assessment include stability (both drug substance and drug product), maximum solubility and suitability for subcutaneous administration, viscosity at high concentrations, tendency toward unwanted microheterogeneity, and compatibility with a platform purification process. Recognizing that the value of many of these attributes does not manifest itself until late in a product's life cycle, early intervention based on the feasibility assessment prior to the initiation of clinical trials is a prudent approach to accommodate manufacturing objectives.

Several companies are evaluating production hosts other than mammalian cells, including transgenic animals or plants, recombinant yeast engineered to express the appropriate glycosyltransferases and thus provide natural oligosaccharides, and full-length mAbs from Escherichia coli. It is unlikely that the purification platform developed for mAbs expressed in mammalian cells will transfer cleanly to mAbs produced by these alternate hosts. The up-front 
clarification and capture steps may be most affected by the change in the nature of the feedstream, yet the polishing and final UF/DF steps will probably be largely unaffacted. The assurance of viral safety will not be an issue for mAbs derived from microbial sources, yet may be a concern for those from transgenic hosts (even plants, if they are grown in an uncontrolled environment).

The dynamic binding capacity for Protein A resins is currently $30-50 \mathrm{~g} / \mathrm{L}$, which offers significant advantages for the processing of high-titer feedstreams when compared to older Protein A media that had capacities at or below $20 \mathrm{~g} / \mathrm{L}$. Increasing the dynamic binding capacity to $80-100 \mathrm{~g} / \mathrm{L}$, to levels commensurate with ion-exchange resins, would result in even smaller in-process pools, reduced buffer consumption, and potentially lower COGs. Although the practical limit to Protein A resin capacity may fall short of $100 \mathrm{~g} / \mathrm{L}$, product capture using ion-exchange resins could offer even higher capacities, albeit probably at the expense of general applicability and streamlined development of Protein A chromatography for product capture.

There would be a great advantage in the development of high-flux, highcapacity parvovirus filters, which would significantly decrease COGs and increase productivity. Reusable virus filters could also significantly affect COGs. For a process with a nonfouling feedstream and control strategy using consistent process flux decay and clean water flux restoration as important process parameters, combined with a validated post-use integrity test, companies may consider developing validation packages that support virus filter reuse. Technology to inactive viruses (heat or ultraviolet irradiation) that could potentially substitute for $\mathrm{VF}$ or $\mathrm{pH}$ inactivation would entail capital costs for new equipment, and could result in scale-up issues.

A major benefit to COGs and manufacturing flexibility could be gained by sharing Protein A resins and membranes between products. Commercial facilities would be able to use resins and membranes to their full lifetime and to enjoy reduced plant changeover losses. For clinical production, the impact on development costs would be even greater-tens of millions of dollars are spent each year on clinical Protein A resins that are used just a few times and then sent to a warehouse, many never to be used again. Accurate and sensitive methods to evaluate product-specific carryover are necessary to ensure that the risk of cross contamination of products is minimized. The testing methods would be combined with a concurrent validation strategy providing direct evidence that the resin had minimal product carryover, perhaps to the standard used for the processing of small molecules using shared compounding equipment (one thousandth of a dose carryover limit). This approach would likely be established on a company-by-company basis, since the testing methods and ability to clean the resin between products would be functions of the unique combination of product characteristics, resin type, and regeneration scheme.

The extent to which Process Analytical Technology (PAT) will be applied to $\mathrm{mAb}$ purification is a controversial topic (34). It seems unlikely that an inherently robust platform process, as demonstrated by process 
characterization for several different $\mathrm{mAbs}$, would require many unit operations using PAT. One potential example of PAT is control of the Protein A column load capacity through the direct measurement of product losses during the load cycle, allowing maximum column loading with each cycle and accommodating changes in dynamic binding capacity with increased column lifetime (23). Alternatively, control could be managed by periodic off-line measurements that would risk little product yield given a slow decline in column capacity. Another solution would be to set the column's load capacity at a more conservative level, which might require only an additional Protein A cycle to process a batch and would not need any measurements or control strategies whatsoever.

\subsection{CONCLUSIONS}

In the more than 20 years since the licensure of the first mAb, antibody purification technology and processes have matured significantly. Platform processes have codified both process designs and development activities at many companies and will have an even more significant impact on the industrialization of commercial mAb production as more mAbs are produced in multiproduct facilities. Advances in process understanding have combined with the introduction of high-performance separation media to enable the rapid definition of increasingly productive processes that yield very pure drug substances.

Through the cumulative impact of high-titer cell culture processes, streamlined purification processes preventing plant productivity bottlenecks, and flexible operations enabling ready transfer of processes between production plants, mAbs will emerge as a special class of therapeutics and will become the least expensive biologics to manufacture, exploiting economies of scale, manufacturing flexibility (both within and between facilities), and common supply-chain elements. This shift may open up the potential for new clinical indications for mAbs, e.g., for the treatment of disease indications that may require high doses and long-term administration, and may affect large patient populations. The drug substance supply for a blend of both blockbuster as well as smaller, niche products could be accommodated by flexible sourcing options afforded by both internal and contract manufacturing, provided that the processes used are based on a common design that is readily transferable to plants configured to run all subtle variations of a standard $\mathrm{mAb}$ purification process.

In one possible future, there will be an excess of cGMP mAb production capacity in the global supply network. All plants will use homologous purification processes based on current platforms that have been updated with modern separation media and refined process designs. This supply network would mirror a decades-long period in the history of global IgIV production, during which there were multiple plasma processing plants worldwide, all of which 
ran slight variations of the Cohn process. Although each plant had slightly different designs, production capacities, and product mixes, the plants were generally more similar than different. The maturation of recombinant $\mathrm{mAb}$ processing (both upstream and downstream), combined with the substantial advances in separation technology from the 1950s to the twenty-first century, makes this future state a distinct possibility.

\subsection{ACKNOWLEDGMENTS}

The authors would like to thank Linda Khym for her assistance in preparing this chapter.

\subsection{REFERENCES}

1. Siegel, J. (2005). The product: All intravenous immunoglobulins are not equivalent. Pharmacotherapy 25, 78S-84S.

2. Johnston, A., Adcock, W. (2000). The use of chromatography to manufacture purer and safer plasma products. Biotechnology and Genetic Engineering Reviews 17, 37-70.

3. Lebing, W., Remington, K.M., Schreiner, C., Paul, H.-I. (2003). Properties of a new intravenous immunoglobulin (IGIV-C, 10\%) produced by virus inactivation with caprylate and column chromatography. Vox Sang 84, 193-201.

4. Fahrner, R.L., Knudsen, H.L., Basey, C.D., Galan, W., Feuerhelm, D., Vanderlaan, M., Blank, G.S. (2001). Industrial purification of pharmaceutical antibodies: Development, operation, and validation of chromatography processes. Biotechnology and Genetic Engineering Reviews 18, 301-327.

5. Shukla, A., Hubbard, B., Tressel, T., Guhan, S., Low, D. (2007). Downstream processing of monoclonal antibodies-Application of platform approaches. Journal of Chromatography B 848, 28-39.

6. Ghose, S., Hubbard, B., Cramer, S.M. (2007). Binding capacity differences for antibodies and $\mathrm{Fc}$-fusion proteins on protein A chromatographic materials. Biotechnology and Bioengineering 96, 768-779.

7. Kelley, B.D., Switzer, M., Bastek, P., Kramarczyk, J., Molnar, K., Yu, T., Coffman, J. (2008). High-throughput screening of chromatographic separations. 4. Ion-exchange. Biotechnology and Bioengineering 100, 950-963.

8. Kelley, B.D., Tobler, S.A., Brown, P., Coffman, J., Godavarti, R., Iskra, T., Switzer, M., Vunnum, S. (2008). Weak partitioning chromatography for anion exchange purification of monoclonal Antibodies. Biotechnology and Bioengineering 101, 553-566.

9. Low, D., O'Leary, R., Pujar, N.S. (2007). Future of antibody purification. Journal of Chromatography $B$ 848, 48-63.

10. Ladisch, M.R., Kohlmann, K.L. (1992). Recombinant human insulin. Biotechnology Progress 8, 469-478.

11. O'Leary, R. (2003). Post-approval changes: A necessary evil in the product lifecycle. Recovery of Biological Products XI, Banff, Alberta, Canada, 2003. 
12. Kelley, B.D., Tannatt, M., Magnusson, R., Hagelberg, S., Booth J. (2004). Development and validation of an affinity chromatography step using a peptide ligand for cGMP production of factor VIII. Biotechnology and Bioengineering 87, 400-412.

13. Kempken, R., Preissmann, A., Berthold, W. (1995). Assessment of a disc stack centrifuge for use in mammalian cell separation. Biotechnology and Bioengineering 46, 132-138.

14. Bender, J., Wolk, B. (1998). Putting a spin on CHO harvest: Centrifuge technology development. ACS Meeting, Boston, MA, 1998.

15. Shpritzer, R., Vicik, S., Orlando, S., Acharya, H., Coffman, J.L. (2006). Calcium phosphate flocculation of antibody-producing mammalian cells at pilot scale. Abstracts of Papers, 232nd ACS Meeting, San Francisco, CA, 2006.

16. Yigzaw, Y., Piper, R., Tran, M., Shukla, A.A. (2006). Exploitation of the adsorptive properties of depth filters for host cell protein removal during monoclonal antibody purification. Biotechnology Progress 22, 288-296.

17. Roque, A.C.A., Silva, C.S.O., Taipa, M.A. (2007). Affinity-based methodologies and ligands for antibody purification: Advances and perspectives. Journal of Chromatography A 1160, 44-55.

18. Zhou, J.X., Tressel, T. (2006). Basic concepts in Q membrane chromatography for large-scale antibody production. Biotechnology Progress 22, 341-349.

19. Luan, Y.-T. (2006). Defined medium development for high yielding mammalian cell culture processes. BioProcess International Conference \& Exhibition: IBC's 7th International Cell Culture \& Upstream Processing, San Francisco, CA, 2006.

20. Jagschies, G., Grönberg, A., Björkman, T., Lacki, K., Johansson, H.J. (2006). Technical and economical evaluation of downstream processing options for monoclonal antibody (Mab) production. BioPharm International 19 (suppl.), 2-8.

21. Kelley, B.D. (2007). Very large scale monoclonal antibody purification: The case for conventional unit operations. Biotechnology Progress 23, 995-1008.

22. Hagel, L., Jagschies, G., Sofer, G. (2007). Production scenarios. In Hagel, L., Jagschies, G., Sofer, G. (eds.) Handbook of Process Chromatography: Development, Manufacturing, Validation and Economics, 2nd eds., Academic Press, London, pp. 22-39.

23. Brorson, K., Brown, J., Hamilton, E., Stein, K.E. (2003). Identification of protein A media performance attributes that can be monitored as surrogates for retrovirus clearance during extended re-use. Journal of Chromatography A 989, 155-163.

24. Blank, G.S. (2006). Modular validation for BLA process ranges. WCBP CMC Strategy Forum, Bethesda, MD, July 20.

25. FDA (2006). International Committee on Harmonisation. Q8: Pharmaceutical Development.

26. Shepherd, A.J., Wilson, N.J., Smith, K.T. (2003). Characterisation of endogenous retrovirus in rodent cell lines used for production of biologicals. Biologicals 31, 251-260.

27. FDA (1999). International Committee on Harmonisation. Q5a: Viral safety evaluation of biotechnology products derived from cell lines of human or animal origin.

28. FDA Center for Biologics Evaluation and Research (1997). Points to Consider in the Manufacture and Testing of Monoclonal Antibody Products for Human Use. FDA Department of Health and Human Services, Rockville, MD. 
29. Brorson, K., Krejci, S., Lee, K., Hamilton, E., Stein, K., Xu. Y. (2003). Bracketed generic inactivation of rodent retroviruses by low $\mathrm{pH}$ treatment for monoclonal antibodies and recombinant proteins. Biotechnology and Bioengineering 82, 321-329.

30. Breece, T., Mallon, E., Giese, G., Persson, J., Gorrell, J. (2006). Evaluation of chromatography media for dual sourcing using a platform technology. Recovery of Biological Products XII, Litchfield, AZ, 2006.

31. FDA Center for Drugs and Biologics (1984). Points to Consider the Production and Testing of New Drugs and Biologicals Produced by Recombinant DNA Technology. FDA Department of Health and Human Services, Rockville, MD.

32. Griffiths, E. (1997). WHO Expert Committee on Biological Standardization: Highlights of the meeting of October 1996. Biologicals 25, 359-362.

33. Anon (2004). Clearer paradigm sought for biopharmaceutical specifications. The Gold Sheet 38, 9-31.

34. FDA Center for Drug Evaluation and Research (2004). Pat-A Framework for Innovative Pharmaceutical Development, Manufacturing, and Quality Assurance. FDA Department of Health and Human Services, Rockville, MD. 
